\title{
Plaque disclosing agent as a guide for professional biofilm removal: A randomized controlled clinical trial
}

\author{
Magda Mensi ${ }^{1,3}$ (D) | Eleonora Scotti ${ }^{1,3}$ | Annamaria Sordillo ${ }^{1}$ | Raffaele Agosti ${ }^{1}$ | \\ Stefano Calza ${ }^{2}$
}

\author{
${ }^{1}$ Section of Periodontics, School of \\ Dentistry, Department of Surgical \\ Specialties, Radiological Science and Public \\ Health, University of Brescia, Brescia, Italy \\ ${ }^{2}$ Department of Molecular and Translational \\ Medicine, University of Brescia, Brescia, \\ Italy \\ ${ }^{3}$ U.O.C. Odontostomatologia - ASST degli \\ Spedali Civili di Brescia, Brescia, Italy

\section{Correspondence} \\ Magda Mensi, Section of Periodontics, \\ School of Dentistry, Department of Surgical \\ Specialties, Radiological Science and Public \\ Health, University of Brescia, P.le Spedali \\ Civili 1, 25123 Brescia, Italy. \\ Email:magda.mensi@unibs.it
}

\begin{abstract}
Objectives: To evaluate through computer software analysis, the efficacy of the use of a plaque disclosing agent as a visual guide for biofilm removal during professional mechanical plaque removal in terms of post-treatment residual plaque area (RPA).

Methods: Thirty-two healthy patients were selected and randomized in two groups to receive a session of professional mechanical plaque removal with air-polishing followed by ultrasonic instrumentation with (Guided Biofilm therapy-GBT) or without (Control) the preliminary application of a plaque disclosing agent as visual guide. The residual plaque area (RPA) was evaluated through re-application of the disclosing agent and computer software analysis, considering the overall tooth surface and the gingival and coronal portions separately.

Results: A statistically and clinically significant difference between treatments is observed, with GBT achieving an RPA of $6.1 \%$ (4.1-9.1) vs $12.0 \%(8.2-17.3)$ of the Control on the Gingival surface and of 3.5\% (2.3-5.2) vs 9.0\% (6-13.1) on the Coronal, with a proportional reduction going from $49.2 \%(P$-value $=.018)$ on the former surface to more than $60 \%(P$-value $=.002)$ on the latter.

Conclusion: The application of a plaque disclosing agent to guide plaque removal seems to lead to better biofilm removal.

KEYWORDS

air-polishing, biofilm, dental biofilm, oral hygiene, plaque disclosing
\end{abstract}

\section{1 | INTRODUCTION}

The oral cavity is the natural habitat of a heterogeneous population of bacteria. ${ }^{1}$ Both soft and hard surfaces are the substrate where microorganisms adhere and grow, forming the oral biofilm. ${ }^{1,2}$

Biofilm quantity and complexity increase with time and affect the environment, leading to the development of caries, gingivitis ${ }^{2,3}$ and periodontitis, ${ }^{4}$ according to individual susceptibility and risk factors. Vice versa, the environment and local factors can influence the growth of biofilm, leading to its diversification in distinct areas even of the same tooth. ${ }^{2}$

The regular disruption of biofilm through professional mechanical plaque removal and home oral hygiene is a critical point in the prevention of caries and periodontal disease. ${ }^{5-8}$ Professional mechanical plaque removal in cariology involves biofilm and calculus removal from the supra-gingival area while, in periodontology, it extends to the sub-marginal space. ${ }^{8}$ While manual and ultrasonic instrumentation constitutes the traditional professional mechanical plaque removal procedure, air-polishing with low-abrasiveness 
powder is of more recent introduction and is regarded as a promising way to manage supra- and sub-gingival biofilm, with advantages in terms of time and comfort. ${ }^{9-11}$ The clinical results during periodontal maintenance therapy are comparable with the ones obtained via traditional scaling and root planing. ${ }^{10,12}$

Regardless of the instruments used and time, complete biofilm removal from hard surfaces is hardly achievable. ${ }^{13,14}$ The aim of professional mechanical plaque removal is to keep the bacterial population below the "critical mass," that is where an equilibrium with the host can exist. ${ }^{15}$ Being individual tolerance highly variable and non-definable, ${ }^{16}$ it is essential to keep oral biofilm level as low as possible.

Oral biofilm is mostly colourless. Disclosing tablets and liquids can allow its visualization for clinical and research purposes. ${ }^{17}$ Disclosing is proven to ensure complete cleaning of molar occlusal surfaces before sealants, ${ }^{18}$ increase biofilm control on dentures, ${ }^{19}$ allow a more efficient debridement of root surfaces during periodontal resective surgery ${ }^{20}$ and, in case of agents able to identify acid-producing bacterial populations, assist in caries risk assessment. ${ }^{21}$ The ability to see the biofilm can also improve patients education and motivation and guide their self-performed oral hygiene. ${ }^{22-24}$ To date, no studies are available involving the use of plaque disclosing agents as a guide for the clinician during professional mechanical plaque removal.

In the research field, application of disclosing agents and subsequent photograph software analysis can be used as an advanced plaque quantification tool, ${ }^{25,26}$ allowing to overcome classic plaque indices limitations, such as variability between different examiners and centres. ${ }^{17}$ Comparisons between planimetric methods and conventional indices show that the former ones are more precise, objective, sensitive and reproducible, and can detect even small changes in plaque area. ${ }^{17,25,26}$

The aim of the present study was to evaluate through computer software analysis-also known as planimetric plaque analysis-the efficacy of the use of a plaque disclosing agent as a visual guide for biofilm removal during professional mechanical plaque removal and compare it with the same procedure without any visual aid in terms of post-treatment residual plaque area (RPA).

\section{2 | STUDY POPULATION AND METHODOLOGY}

\section{1 | Study design and population}

The present study was a single-blinded, randomized, controlled clinical trial with 2 parallel groups, conducted in accordance with the Helsinki Declaration and approved by the Ethics Committee of Spedali Civili di Brescia, protocol number 2636.

Thirty-two (32) systemically healthy subjects were selected from the population afferent to the Dental School "Clinica Odontoiatrica Lidia Verza," University of Brescia, Department of Radiological Science and Public Health, within the ASST Spedali
Civili di Brescia, Department of Odontostomatology (Brescia, Italy). The patients showed no sign of periodontal disease but presented a Plaque Index ${ }^{27}$ (PI) exceeding $25 \%$ and required professional oral care (professional mechanical plaque removal and oral hygiene instructions).

The inclusion criteria were as follows:

- Systemically healthy patients

- No missing anterior teeth

- $\geq 18$ years of age

- $\mathrm{Pl}^{27}>25 \%$

- No smoking or smoking <10 cigarettes/d

- Need for professional oral care (professional mechanical plaque removal and oral hygiene instructions)

The exclusion criteria were as follows:

- Presence of periodontal disease, defined as $>3 \mathrm{~mm}$ of clinical attachment loss at any site

- Presence of fix retainers

- Presence of orthodontic appliances

- Prosthetic rehabilitation of anterior sextants

- Pregnant and lactating patients

- Unwillingness to undergo the proposed protocol

All the participants signed written informed consent before the beginning of the study.

\section{2 | Intervention}

A total of 32 eligible subjects were randomized in two groups: the test group received a session of professional mechanical plaque removal guided by the application of a plaque disclosing agent as a visual guide for the clinician (named by the authors Guided Biofilm Therapy-GBT), while the Control group received the same professional mechanical plaque removal procedure without any visual aid.

After the placement of a lips and cheeks retractor (OptraGate ${ }^{\circledR}$, Ivoclar Vivadent) and the collection of Plaque Index (PI), ${ }^{27}$ the patients were allocated to one of the groups (GBT or Control) via randomization list and numbered opaque envelopes. In the GBT group, the plaque disclosing agent (MIRA-2-TON ${ }^{\circledR} 60 \mathrm{~mL}$ bottle, HAGER WERKEN) was then applied by the operator with a micro-brush to cover the entire tooth surface and thoroughly rinsed with water (Figure 1).

In both groups, professional mechanical plaque removal was performed with an air-polishing device (Air-flow Master Piezon ${ }^{\circledR}$ EMS). The protocol follows the glycine powder air-polishing (GPAP) principles outlined by Flemmig et $\mathrm{al}^{11}$ but with the use of the more recently introduced erythritol powder (PLUS powder ${ }^{\circledR}$ EMS) and involves supra-gingival and sub-gingival biofilm removal via air-polishing as the first step. The erythritol powder was preferred due to its similar physical properties, the clorhexidine content $(0.3 \%)$ and the 

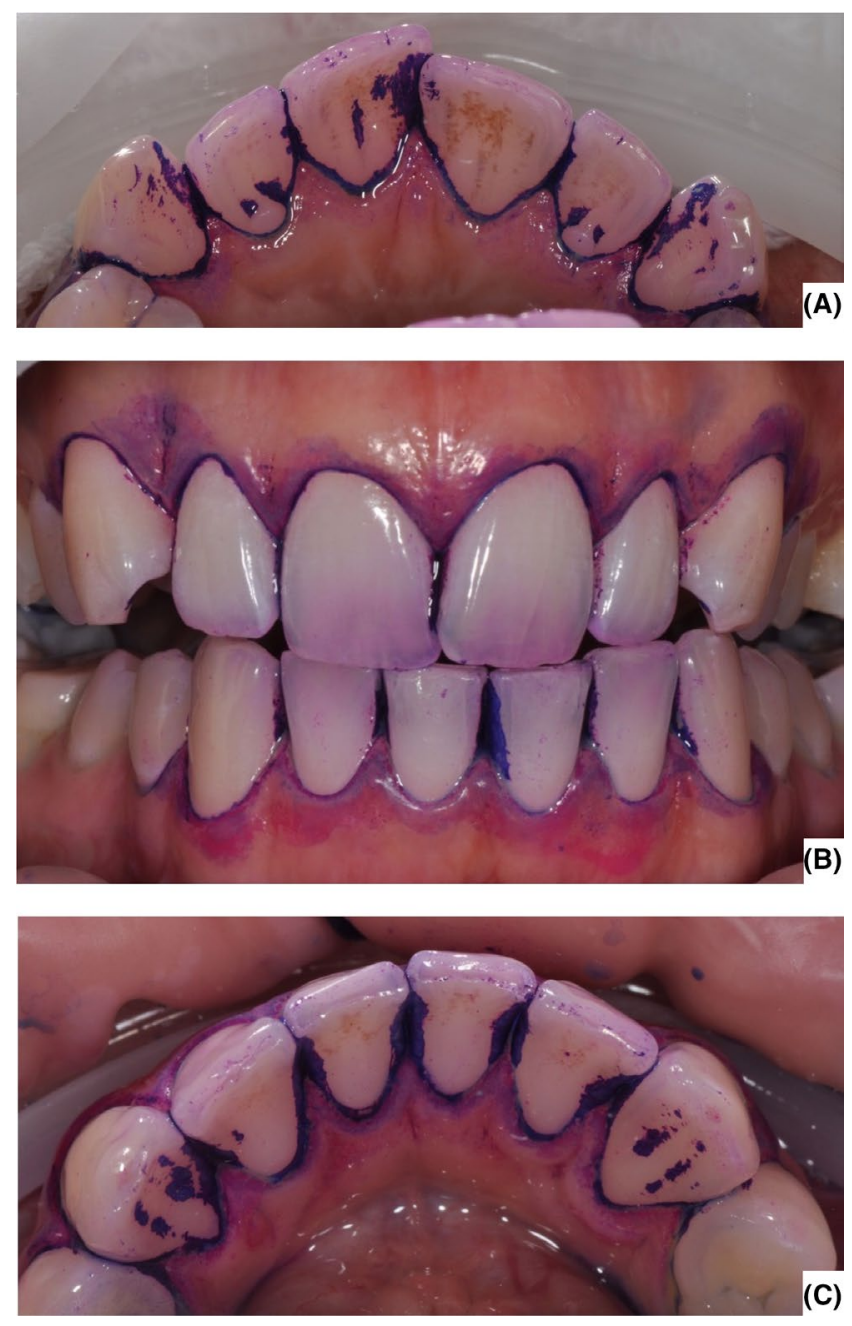

FIGURE 1 Application of the disclosing agent before the therapy, palatal view (A), buccal (B) and lingual (C)

recent evidence of its safety and efficiency. ${ }^{10,12}$ At completion, calculus removal was performed with a piezoceramic device (Air-flow Master Piezon ${ }^{\circledR}$ EMS) and a slim tip (PS ${ }^{\circledR}$ EMS) only if hard deposits are present.

In the GBT group, the session ended when no visible disclosing agent was left (Figure 2), while in the Control group it ended when the clinician was confident biofilm removal was complete. In both groups, the disclosing agent was re-applied and photographs were taken to locate the residual biofilm (Figure 3).

Because of difficulties in the standardization of intra-oral photography and computer analysis limitations, only the second and fifth sextants were considered in this study. A white colour-calibration target was used in conjunction with mirrors to collect buccal, lingual and palatal photographs of the second and fifth sextants. An extra-oral camera was used (Nikon D90 with AF-S VR Micro-Nikkor $105 \mathrm{~mm}$ f/2.8G IF-ED) with standardized camera settings (focus distance $40 \mathrm{~cm}$ to subject, f/36, 1/160s) and flash settings (Metz Mecablitz 15 MS-1 Digital Flash Anular, 1/8 flash power for the buccal shots and 1/4 flash power for the lingual and palatal). All the photographs were taken by the same expert operator.
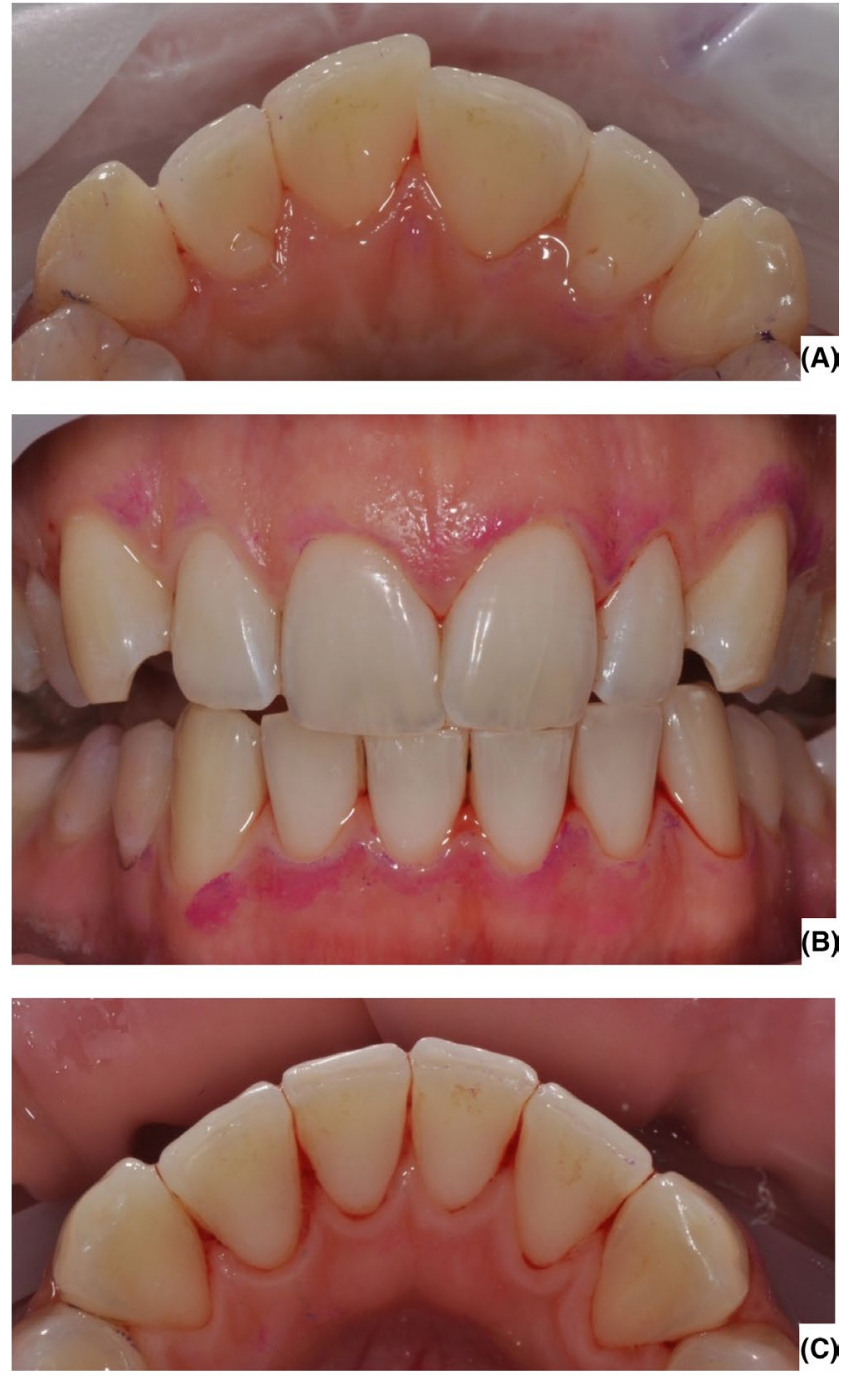

FIGURE 2 Post-therapy, palatal view (A), buccal (B) and lingual (C)

\section{3 | Image analysis}

The clinical photographs were processed by an operator blinded to the group allocations through ImageJ software (National Institutes of Health). The area covered by the disclosing agent (residual plaque area-RPA) was calculated as $\%$ of the total teeth area.

Image analysis started with the manual selection of the following surfaces:

1. Entire clinical crown, from incisal to gingival margin, excluding soft tissues and background (Figure 4) -named Overall;

2. Gingival third of the clinical crown-named Gingival.

3. Coronal two-thirds of the clinical crown-named Coronal

The area of interest was selected and cropped with particular care along the gingival margin and in the interproximal areas, to avoid the inclusion of the soft tissues. The sections were first converted to RGB-stacks and then to greyscale (Figure 5), obtaining per each image three different elaborations based on the red, green and 

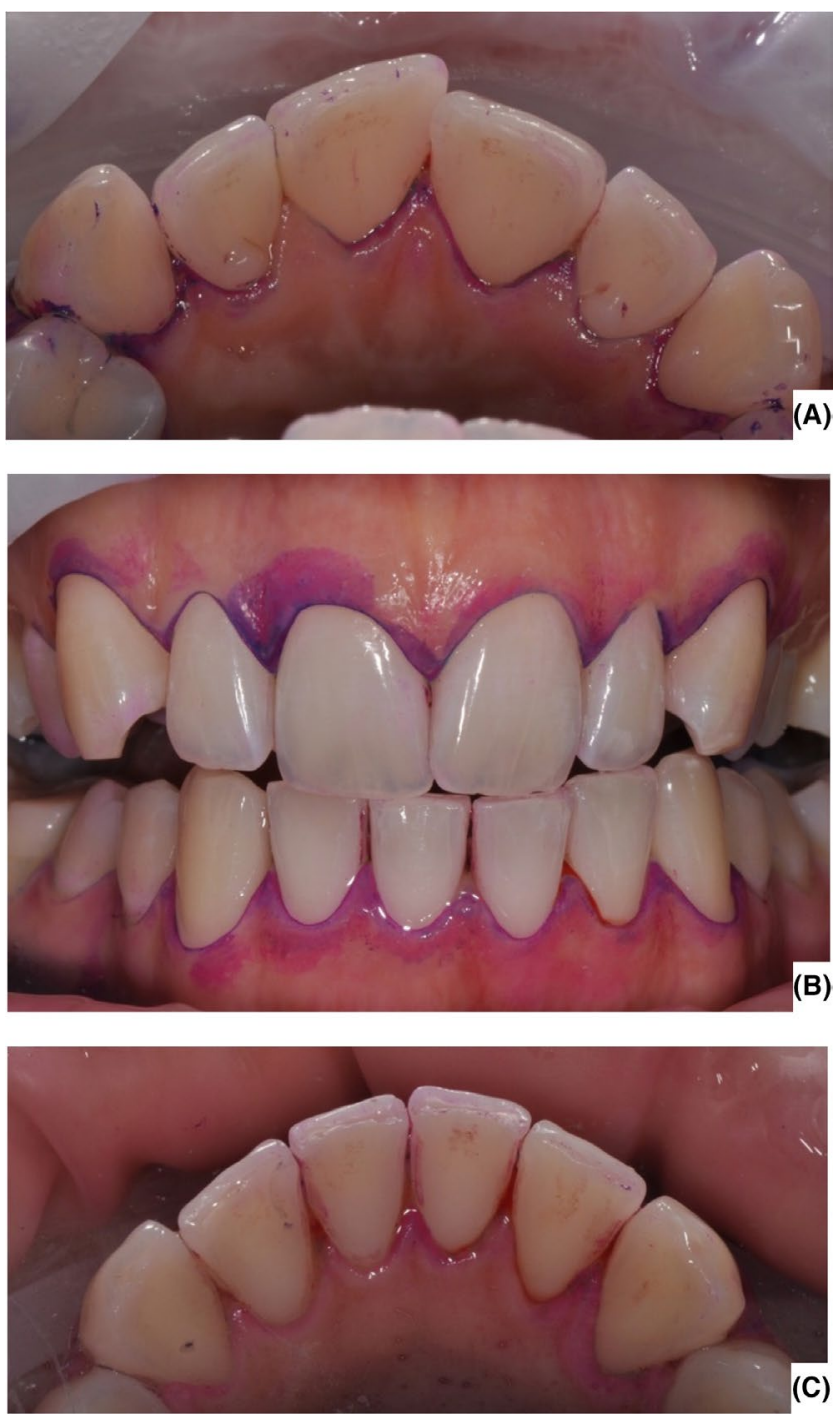

FIGURE 3 Re-application of the disclosing agent. Palatal view (A), buccal (B) and lingual (C)

blue channels. The green-channel elaborations were chosen for the next step, as green is the colour that better highlights the pink-purple tint of the plaque disclosing agent, shown as dark-grey/black. Though the colour threshold selection function, the range within the 0-255 greyscale corresponding to the disclosing agent was set, and the pixel-based percentage (hereafter indicated percentage of area with residual plaque) of the disclosing-coloured areas was calculated (Figure 6).

\section{4 | Statistical analysis}

The sample size was computed assuming a two independent group comparison based on t test allowing for different variances (Welch's test). We assumed $5 \%$ and $10 \%$ residual plaque (\% of plaque are over total teeth inspected area), respectively, and a $60 \%$ coefficient of variation for both groups. Considering an $80 \%$ power and a $5 \%$ significance level, we computed a total sample size of $N=32$ (16
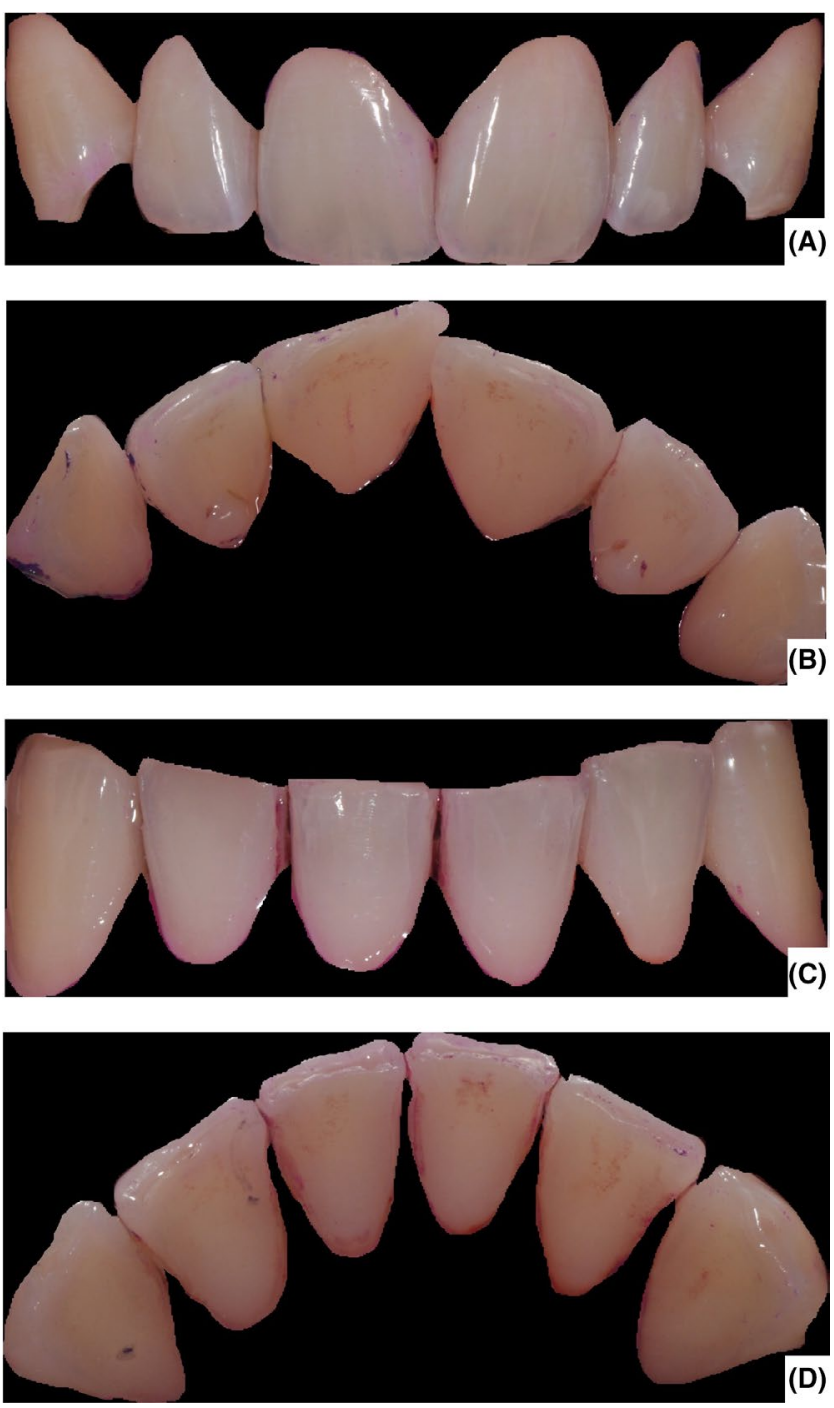

FIGURE 4 Sections of photographs taken after therapy and application of plaque detector. The entire gingival portion was removed to show only the dental crown (Overall surface). Palatal view $(A)$, upper buccal $(B)$, lower buccal $(C)$ and lingual(D)

for each group). To allow for potential deviations from normality assumption for percentages, we also computed sample size using a Wilcoxon-Mann-Whitney simulation based on 2000 Monte Carlo samples from the null distributions (with parameters as specified above) achieving a consistent (software: PASS 13). Patients were randomized using a computer-generated randomization list. The random allocation sequence was generated with uninformative labels ( $\mathrm{A}$ and $\mathrm{B}$ ) and using block randomization algorithm (block size $=4$ ). All data analyses were carried out according to a pre-established analysis plan by a biostatistician blinded to group allocation. The percentage of area with residual plaque was modelled at tooth level using a linear mixed models (LMM) using a random intercept model with Patient as a random component to account for data clustering. Residual area values were transformed on logit prior to modelling. Estimated PI at baseline was computed after aggregation within patients, that is PI was computed as the number of sites with plaque within the subject. This was modelled using a GLM with negative 

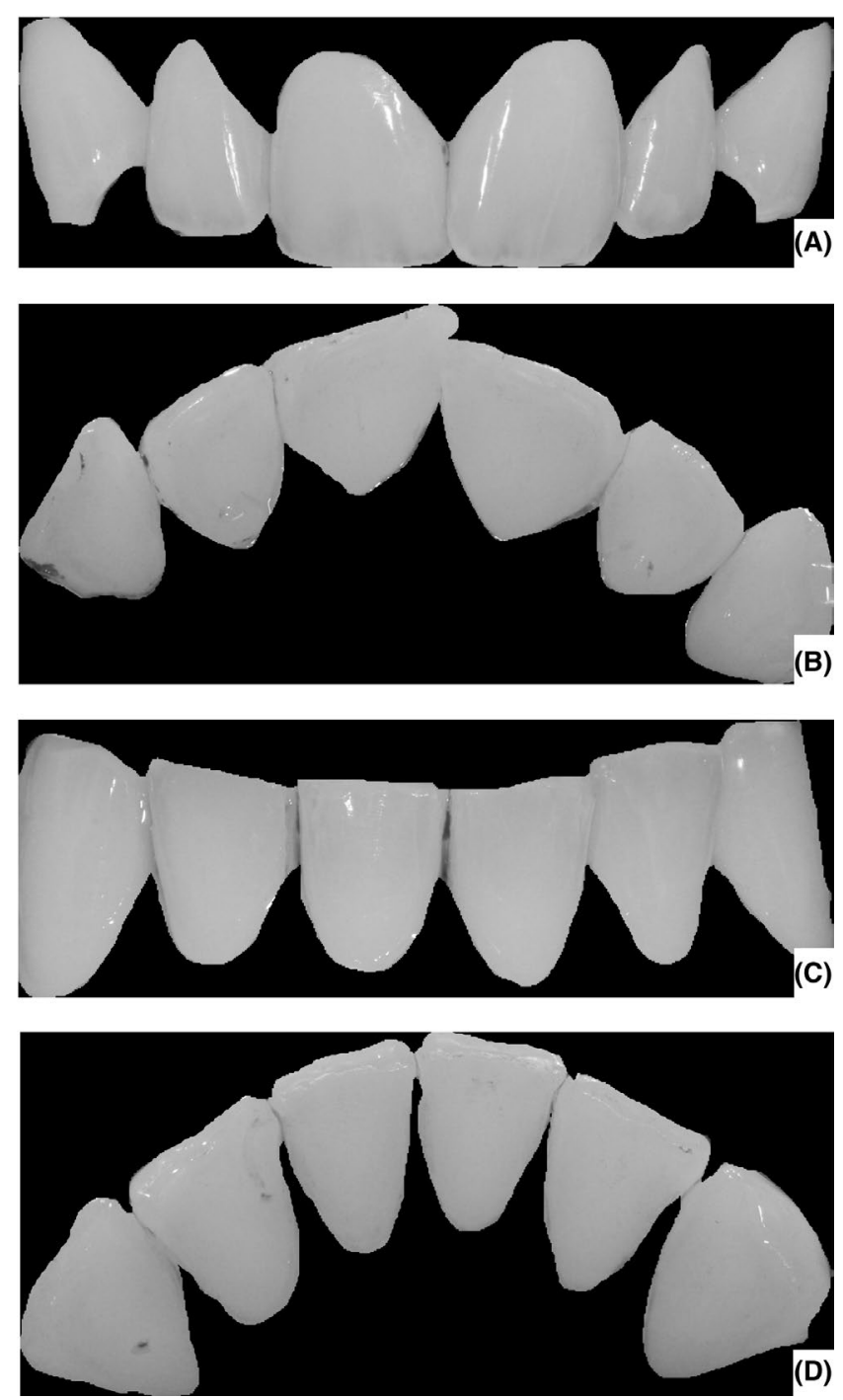

FIGURE 5 Sections previously obtained converted to grayscale on an RGB basis. Show respectively palatal view (A), upper buccal (B), lower buccal (C) and lingual(D)

binomial family and using the total number of evaluated sites within the subject as an offset. PI estimates were adjusted for Gender and Smoking status. All the analyses were performed using R (version 3.5.2), assuming a $5 \%$ level of significance.

\section{3 | RESULTS}

Results are reported as estimate and 95\% confidence interval. Proportional variation is expressed as the variation going from Control to GBT expressed as a percentage relative to Control starting value.

Table 1 reports the PI estimates at baseline for both treatments showing a substantial homogeneity between groups. Because of the design of the study, especially the intervention in the Control group, it was not possible to use the same planimetric analysis method for initial plaque quantification.
The residual plaque area (RPA) measurements for both treatments on the Gingival and the Coronal surfaces are presented in Table 2 and Graphic 1. A statistically and clinically significant difference between treatments is evident in both location, with GBT achieving a lower RPA, with a proportional reduction going from $49.2 \%(P$-value $=.018)$ on the Gingival surface to more than $60 \%$ $(P$-value $=.002)$ on the Coronal surface. Overall, we also observed a higher RPA on the Gingival surface compared with the Coronal one.
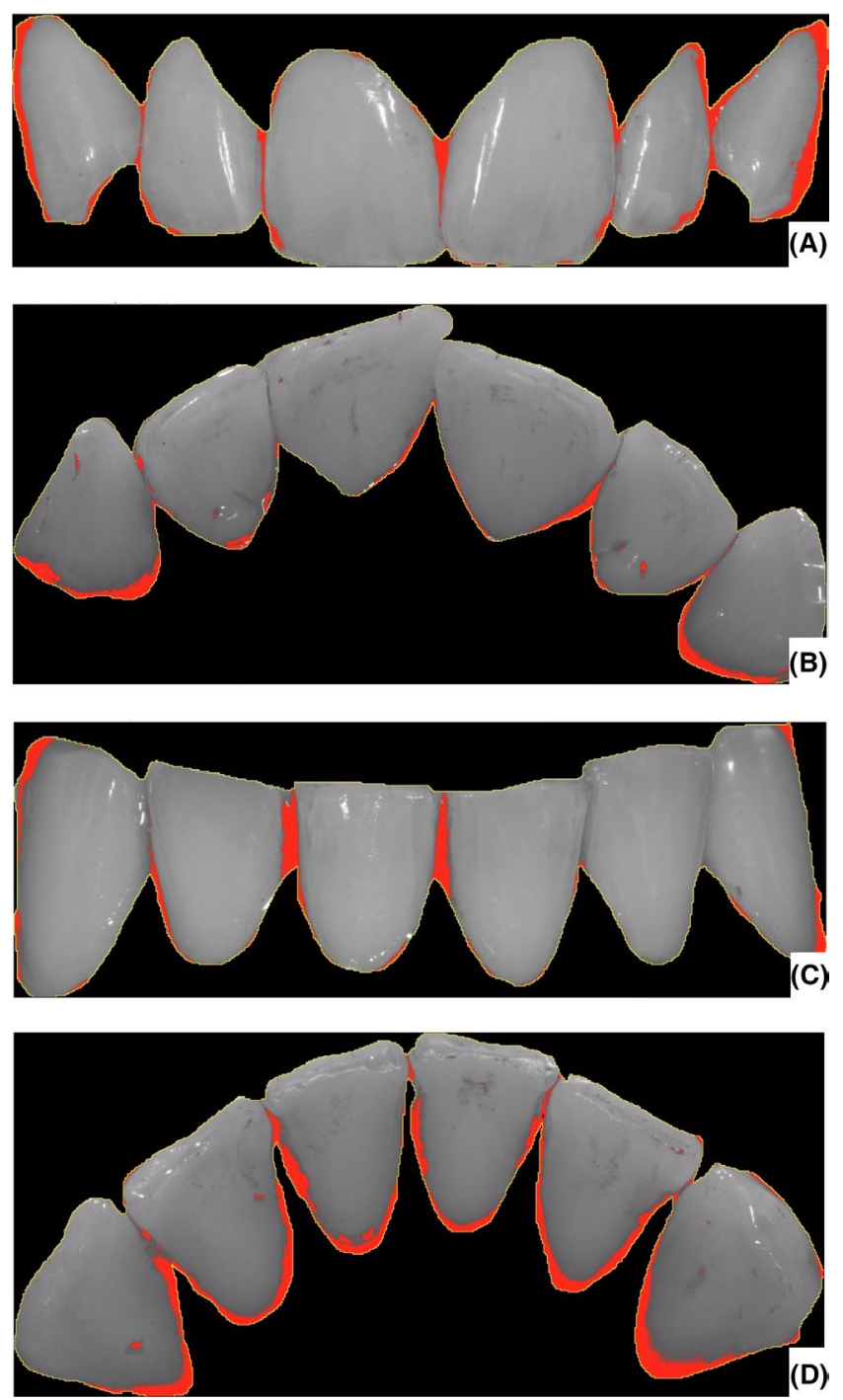

FIGURE 6 The Threshold command is applied to the processing of Figure 5, which allows you to select the desired colour range and highlight it, in order to calculate the area. The clippings show the area subtended by the plaque detector (highlighted in red) on the palatal view (A), upper buccal (B), lower buccal (C) and lingual(D)

TAB LE 1 Baseline plaque index, according to O'Leary $(1972)^{27}$

\begin{tabular}{ll} 
Group & Plaque index \\
GBT & $83.1 \%(73.2 \%-94.5 \%)$ \\
Control & $82.8 \%(71.2 \%-96.2 \%)$ \\
\hline -value & .96 \\
\hline
\end{tabular}




\begin{tabular}{llllll} 
& GBT & Control & $\begin{array}{l}\text { proportional } \\
\text { variation }\end{array}$ & $\begin{array}{l}\text { absolute variation } \\
\text { (Control - GBT) }\end{array}$ & $\begin{array}{l}\text { P- } \\
\text { value }\end{array}$ \\
\hline Gingival & $6.1(4.1-9.1)$ & $12.0(8.2-17.3)$ & 49.2 & 5.9 & .018 \\
\hline Coronal & $3.5(2.3-5.2)$ & $9.0(6-13.1)$ & 61.1 & 5.5 & .002 \\
\hline Total & $4.8(3.3-6.8)$ & $10.3(7.3-14.3)$ & 54.0 & 5.5 & .003 \\
\hline
\end{tabular}

TABLE 2 RPA (residual plaque area) after PMPR session, considering the overall tooth surface and Gingival and Coronal surfaces separately. All values are reported as percentages
Table 3 shows the RPA on the Overall surface, considering upper buccal, palatal, lower buccal and lingual areas separately. In the upper buccal area, the RPA value of GBT is $3.4 \%$ compared with $5.9 \%$ of Control with a proportional variation equal to 43.4 with a $P$-value of .098. In the lower buccal portion, the RPA value of GBT is $5.1 \%$ compared with $11.1 \%$ of Control, with a proportional variation of 54.3 and a P-value of .020 . In the palatal portion, the RPA value of GBT is to $3.5 \%$ compared with $7.6 \%$ of Control, with a proportional variation of 53.4 and a $P$-value of .026. Finally, in the lingual portion, the RPA value of GBT is $4.8 \%$ compared with $12.5 \%$ of Control, with a proportional variation of 61.5 and a $P$-value of .05 . All the subdivisions show a higher efficacy of GBT with proportional variations $>50 \%$ except for the buccal portion, that does not reach statistical significance $(P$-value $=.098)$.

Table 4 shows the RPA on the Gingival surface, considering upper buccal, palatal, lower buccal and lingual areas separately. In the upper buccal portion, the RPA value of GBT is to $4.9 \%$ compared with $8.5 \%$ of Control with a proportional variation equal to 42.1 with a $P$-value of .102. In the lower buccal portion, the RPA value of GBT is $5.3 \%$ compared with $9.9 \%$ of Control, with a proportional variation of 46.2 and a $P$-value of .063. In the palatal portion, the RPA value of GBT is $4.3 \%$ compared with $10.5 \%$ of Control, with a proportional variation of 58.7 and a P-value of .009. Finally, in the lingual portion, the RPA value of GBT is $8 \%$ compared with the $14.8 \%$ of Control, with a proportional variation of 46.0 and a $P$-value of .054

Table 5 shows the RPA on the Coronal surface, considering upper buccal, palatal, lower buccal and lingual areas separately. In the upper buccal portion, the RPA of GBT is $2.1 \%$ compared with $3.7 \%$ of Control with a proportional variation equal to 43.0 with a $P$-value of .256. In the upper buccal portion, the RPA value of GBT is $4.0 \%$ compared with $10.4 \%$ of Control, with a proportional variation of 61.8 and a $P$-value of .044. In the palatal portion, the RPA value of GBT is $1.8 \%$ compared to $4.3 \%$ of Control, with a proportional variation of 59.0 and a $P$-value of .073 . Finally, in the lingual portion, the RPA value of GBT is $2.4 \%$ compared with $10.6 \%$ of Control, with a proportional variation of 77.3 and a P-value of .003 .

\section{4 | DISCUSSION}

The present study represents the first of a series aimed to investigate and validate the concept of Guided Biofilm Therapy (GBT), whose significant novelties are the use of plaque disclosing as a visual guide and the predominant use of an air-polishing device for biofilm removal. The choice of the authors is due to the desire to progress towards a minimally invasive professional mechanical plaque removal concept and is supported by the evidence that supra- and sub-gingival air-polishing is safe and conservative on both soft and hard tissues, more time-efficient and more comfortable for the patient. ${ }^{10,28-32}$ Furthermore, it allows reducing the use of ultrasonic/ manual instrumentation to the minimum required to remove hard calculus.

Plaque disclosing through tablets and liquids is a well-known tool to help patients visualize the oral plaque and improve their self-performed hygiene and compliance, both in a professional and home setting. ${ }^{33,34}$ It is also proven to ensure complete cleaning of molar occlusal surfaces before fissure sealing, ${ }^{18}$ to increase biofilm control on dentures ${ }^{19}$ and to allow better debridement of root surfaces during resective periodontal surgery. ${ }^{20}$ In the context of professional oral hygiene, one could assume that the plaque disclosing can be beneficial not only for the patient but also for the clinician as a guide for biofilm removal, allowing immediate feedback, especially for those areas difficult to access and for those individuals at high risk of carious or periodontal pathology. To date, no clinical trials are available to prove the assumption; hence, the present study aimed to measure the potential advantage of the use of a plaque disclosing agent as a visual guide for the clinician during professional mechanical plaque removal, compared with the same treatment without any aid.

The need for detection of small areas of plaque and reproducibility determined our choice to adopt a planimetric plaque analysis method over a clinical plaque index and to express the residual plaque as a percentage of the selected areas (Overall, Gingival and Coronal). Automated planimetric analysis allows a more sensitive and objective plaque localization and quantification when

\begin{tabular}{|lllll|} 
& GBT & Control & $\begin{array}{l}\text { proportional } \\
\text { variation }\end{array}$ & $\begin{array}{l}\text { P- } \\
\text { value }\end{array}$ \\
\hline Upper buccal & $3.4(2.1-5.4)$ & $5.9(3.7-9.5)$ & 43.4 & .098 \\
\hline Lower buccal & $5.1(3.1-8.1)$ & $11.1(7-17.1)$ & 54.3 & .020 \\
\hline Palatal & $3.5(2.2-5.7)$ & $7.6(4.7-11.9)$ & 53.4 & .026 \\
\hline Lingual & $4.8(3-7.7)$ & $12.5(8-19.1)$ & 61.5 & .005 \\
\hline
\end{tabular}

TABLE 3 RPA (residual plaque area) after PMPR session, considering the Overall surface. Upper buccal, palatal, lower buccal and lingual areas are analysed separately. All values are reported as percentages 
TABLE 4 RPA (residual plaque area) after PMPR session, considering the Gingival surface. Upper buccal, palatal, lower buccal and lingual areas are analysed separately. All values are reported as percentages

TABLE 5 RPA (residual plaque area) after PMPR session, considering the Coronal surface. Upper buccal, palatal, lower buccal and lingual areas are analysed separately. All values are reported as percentages

FIGURE 7 Clinical comparison between a GBT and a Control patient after treatment and re-application of the disclosing agent

\begin{tabular}{lllll|} 
& GBT & Control & $\begin{array}{l}\text { proportional } \\
\text { variation }\end{array}$ & $\begin{array}{l}\text { P- } \\
\text { value }\end{array}$ \\
\hline Upper buccal & $4.9(3-7.8)$ & $8.5(5.3-13.2)$ & 42.1 & .102 \\
\hline Lower buccal & $5.3(3.3-8.4)$ & $9.9(6.2-15.3)$ & 46.2 & .063 \\
\hline Palatal & $4.3(2.7-6.9)$ & $10.5(6.6-16.2)$ & 58.7 & .009 \\
\hline Lingual & $8(5-12.5)$ & $14.8(9.5-22.2)$ & 46.0 & .054 \\
\hline
\end{tabular}

\begin{tabular}{llllc|} 
& GBT & Control & $\begin{array}{l}\text { proportional } \\
\text { variation }\end{array}$ & $\begin{array}{l}\text { P- } \\
\text { value }\end{array}$ \\
\hline Upper buccal & $2.1(1.1-4.2)$ & $3.7(1.9-7.3)$ & 43.0 & .256 \\
\hline Lower buccal & $4(2-7.7)$ & $10.4(5.4-19)$ & 61.8 & .044 \\
\hline Palatal & $1.8(0.9-3.5)$ & $4.3(2.2-8.3)$ & 59.0 & .073 \\
\hline Lingual & $2.4(1.2-4.8)$ & $10.6(5.5-19.4)$ & 77.3 & .003 \\
\hline
\end{tabular}
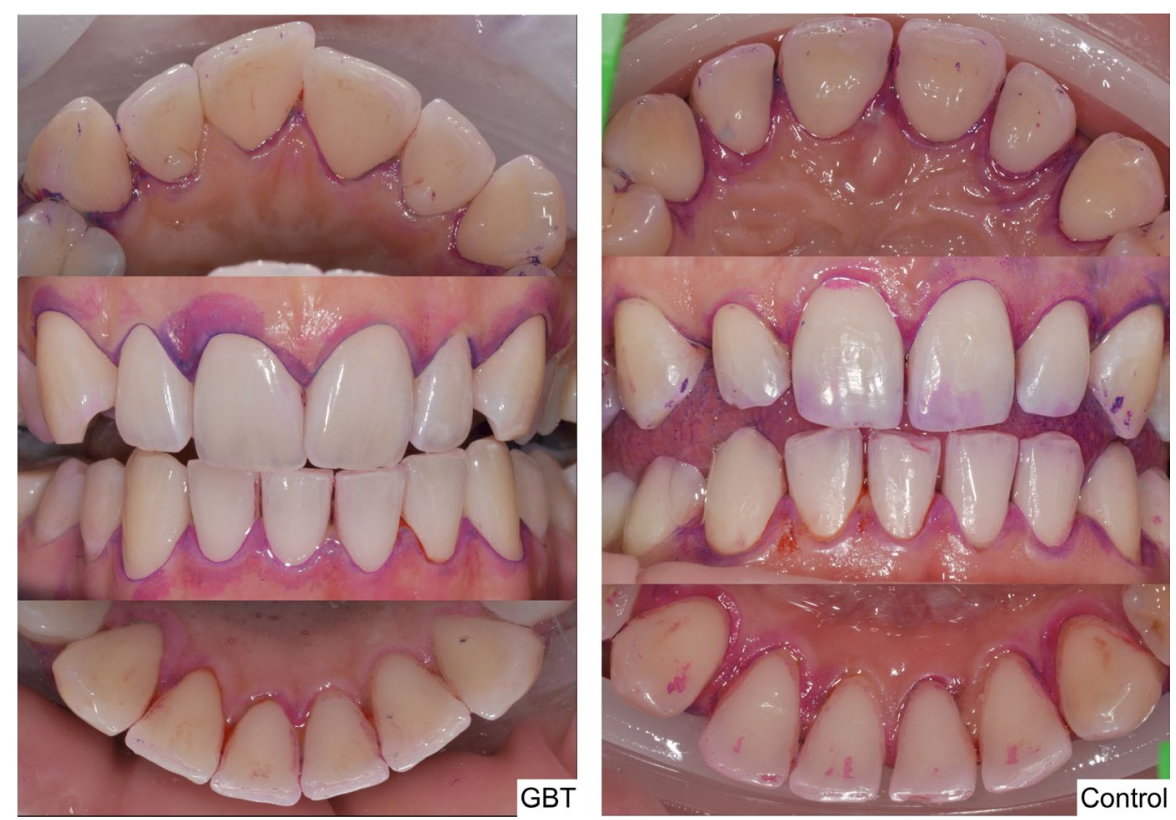

compared to the conventional clinical indices ${ }^{17,25,26,35}$ and has high discriminating power, allowing to detect even minimal changes in plaque area. ${ }^{25}$

While some studies report the use of camera-to-head positioning frames, ${ }^{25}$ in some others the photogrphs are taken freely but with the same focal distance and settings. ${ }^{35}$ In the present study, we decided to use an extra-oral camera and standardized settings. Even if a frame for camera-to-head positioning was not used, we are confident that through the use of the same settings, the same expert operator and the randomization process, the results are accurate and reproducible.

A limitation of the image elaboration process adopted could be the necessity to manually select and cut the teeth areas of the images, eliminating soft tissues and background, with the risk of not being able to identify the gingival margin and papillae accurately. Nevertheless, Smith et al $^{25}$ show that manual selection does not impair the intra- and inter-operator reliability, which is still excellent.
Most importantly, as in Smith et al, ${ }^{25}$ our protocol does not involve manual area tracing of plaque regions, but an automatized colour encoding by the ImageJ software, eliminating human error in the crucial step of plaque and non-plaque areas discrimination.

At baseline (Table 1), both experimental groups show homogeneity of PI. Because of the design of the study, especially the intervention in the control group, it was not possible to use the same planimetric analysis method for initial plaque quantification.

At the end of the professional mechanical plaque removal session, the RPA in the GBT group was significantly lower than in the Control group. An example of results obtained with GBT and Control is shown in Figures 7 and 8, comparing the subjects clinically and via software analysis. When considering the Gingival and Coronal surfaces separately, the GBT group showed, respectively, half and a third of the mean RPA area of the Control group (Table 2, Graphic 1).

The decision to analyse the Gingival portion of the clinical crown separately comes from the fact that biofilm at and below gingival 

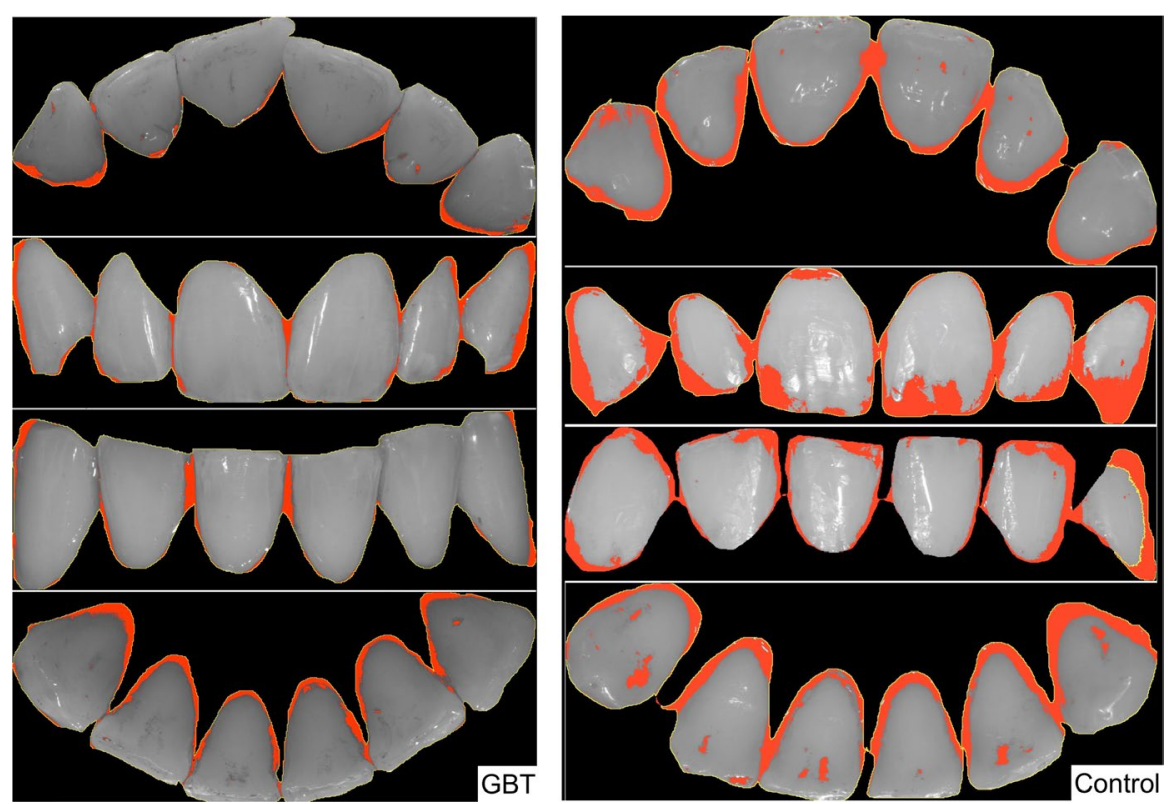

FIGURE 8 Software elaboration of the images in Figure 7, with the plaque disclosing agent highlighted in red

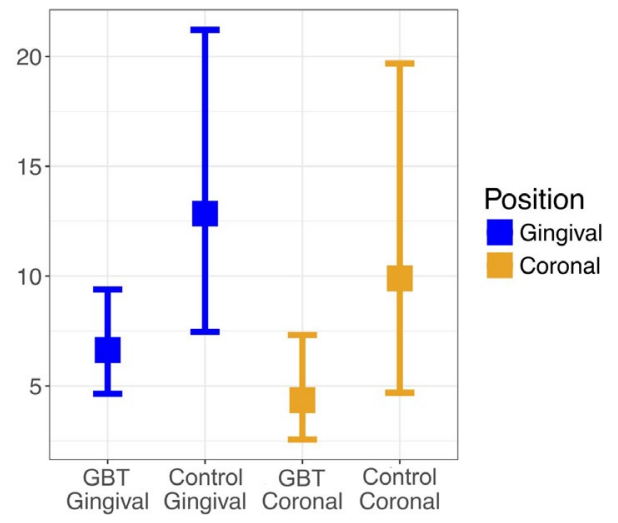

GRAPHIC 1 Estimated average percentages of areas with residual plaque and corresponding 95\% confidence intervals, grouped for treatment and position

margin is considered the most important risk factor for periodontitis $^{36}$; hence, its removal is of significant importance. Interestingly, the GBT group results (Table 2 ) have a confidence interval of $<10 \%$ [6.1 (4.1-9.1)] while the Control group a confidence interval of $<18 \%$ [12 (8.2-17.3)]. Hence, despite showing a small difference in linear percentage points, the GBT procedure gives not only better biofilm removal but also higher inter-patients consistency. This observation can be due to the fact that, being oral biofilm mostly colourless, when professional mechanical plaque removal is performed without a visual aid, it is primarily based on the operator's experience and feeling, adding subjectivity and human error to the procedure. In both groups, plaque removal was better performed on the Coronal surface (Table 2) probably because it is usually an area easier to clean both for the patient and the clinician. Unfortunately, the PI index used at the baseline does not allow us to know how much of the initial plaque was located on the Coronal surface.

Considering the upper buccal, palatal, lower buccal and lingual areas separately, for the Gingival surface (Table 4), GBT performed significantly better than Control only in the palatal surface, but the values for the lower buccal and lingual ones are on the edge of significance. For the Coronal surface (Table 5), GBT seems significantly superior in the lower arch and is on the edge of significance for the palatal area. Further investigations on a bigger sample size would help clarify these findings. The only area never reaching a statistically significant difference between the two groups is the upper buccal. We can assume that plaque disclosing guidance is not of major importance in this area because of direct visibility, better access and bigger size of the teeth. On the other hand, GBT is linked to lower RPA at the palatal side, often requiring indirect vision, and the lingual side with its difficult access and the interposition of the tongue. The same observation was made by Montevecchi et $\mathrm{al}^{20}$ showing that, during resective periodontal surgery, the areas of the root more frequently left unclean after SRP were the distal and lingual, compared to the buccal one.

When interpreting the results from the clinical point of view, both groups showed a satisfactory reduction of plaque at the end of the professional mechanical plaque removal session, being the RPA well below $25 \%$. It is crucial to keep in mind that the patients selected for the present study (adults, systemically and periodontally healthy, no orthodontic appliances and retainers or prosthetic rehabilitation, no crowded teeth) can be considered relatively easy candidates for professional mechanical plaque removal, regardless of the protocol in use. More complex patients can show areas of difficult access and complex surfaces, so one can assume they would benefit even more of a guided mechanical plaque removal procedure and a more significant difference between the two groups would be expected (clinical trials are necessary to verify this assumption). Therefore, selecting our study population, we intentionally excluded possible bias that could favour the GBT process. Furthermore, while in the selected subjects the measured residual plaque might not be relevant for their health status, this might not reflect the clinical reality. The aim of professional mechanical plaque removal is to control and keep the bacterial population below a level where an equilibrium with the host can exist, ${ }^{15}$ but we cannot 
know for sure this threshold of tolerance ${ }^{16}$; hence, the necessity to reduce biofilm as much as possible, especially in highly susceptible patients, such as periodontal, paediatric or orthodontic patients. ${ }^{5,7,14}$

The major limitation of the present study is the fact that the computer analysis protocol chosen can be confidently applied only to anterior teeth, since a validated method to take standardized photographs of posterior areas still does not exist, and intra-oral cameras cannot provide the same level of resolution as the extra-oral ones. Images with dissimilar illumination and angulation can impair the reliability of the software colour analysis and area calculation, hindering the results. Plaque accumulation in the posterior areas is of paramount importance when considering the overall bacterial load and patient's adherence to hygiene instructions, and further investigations are needed to shed some light on this aspect. Furthermore, the software analysis is performed on a 2D image, with limited power to give a real measurement of the interproximal plaque, a crucial area to be kept free-of-plaque in susceptible patients. As mentioned above, another limitation comes from the limited sample size and the type of population selected for the present study, which might not represent the clinical reality for most professional mechanical plaque removal sessions. In future research, it would be of major interest to investigate the role of plaque disclosing in more complex and higher-risk patients and, when the technology will allow it, to perform image software analysis of the posterior areas of the dental arches, where the access for professional mechanical plaque removal is limited. It would also be interesting to conduct the same investigation in conjunction with different protocols of professional mechanical plaque removal, such as the traditional ultrasonic debridement and polishing with a rubber cup and prophylaxis pastes.

In conclusion, within the limitations of the present study, the application of a plaque disclosing agent to guide plaque removal (GBT) seems to lead to better plaque removal, especially in areas of more difficult access.

\section{5 | CLINICAL RELEVANCE}

\section{1 | Scientific rationale for study}

To date, no studies are available involving the use of plaque disclosing agents as a guide for the clinician during professional biofilm removal.

\section{2 | Principal findings}

The application of a plaque disclosing agent seems to lead to better plaque removal, especially in areas of more difficult access.

\section{3 | Practical implications}

The regular use of plaque disclosing agents may improve the level of professionally delivered oral hygiene.

\section{CONFLICT OF INTEREST}

Dr Mensi reports personal fees from ems, personal fees from kulzer, outside the submitted work. Dr Scotti reports personal fees from ems, personal fees from kulzer, outside the submitted work. Dr Sordillo reports personal fees from ems, outside the submitted work. Dr Agosti has nothing to disclose. dr calza has nothing to disclose.

\section{AUTHOR CONTRIBUTIONS}

MM designed the study; SE and AR were the principal investigators; SA wrote the article; CS performed the statistical analysis.

ORCID

Magda Mensi iD https://orcid.org/0000-0001-5807-9338

\section{REFERENCES}

1. Socransky S, Haffajee AD. Periodontal microbial ecology. Periodontol. 2000;2005(38):135-187.

2. Larsen T, Fiehn NE. Dental biofilm infections - an update. APMIS. 2017;125:376-384.

3. Theilade E, Wright WH, Jensen SB, Löe H. Experimental gingivitis in man. II. A longitudinal clinical and bacteriological investigation. J Periodontal Res. 1966;1:1-13.

4. Kinane DF, Attstrôm R, European Workshop in Periodontology group B. Advances in the pathogenesis of periodontitis. Group B consensus report of the fifth European Workshop in Periodontology. J Clinical Periodontol. 2005;32(Suppl. 6):130-131.

5. Tonetti MS, Eickholz P, Loos BG, et al. Principles in prevention of periodontal diseases: consensus report of group 1 of the 11th European Work- shop on Periodontology on effective prevention of periodontal and peri-implant diseases. J Clinical Periodontol. 2015;42(Suppl 16):S5-S11.

6. Sanz M, Beighton D, Curtis MA, et al. Role of microbial biofilms in the maintenance of oral health and in the development of dental caries and periodontal diseases. Consensus report of group 1 of the joint EFP/ORCA workshop on the boundaries between caries and periodontal diseases. J Clinical Periodontol. 2017;44(S18):5-11.

7. Needleman I, Nibali L, Di lorio A. Professional mechanical plaque removal for prevention of periodontal diseases in adults - systematic review update. J Clin Periodontol. 2015;42(Suppl. 16):S12-S35.

8. Figuero E, Nóbrega DF, García-Gargallo M, et al. Mechanical and chemical plaque control in the simultaneous management of gingivitis and caries: a systematic review. J Clin Periodontol. 2017;44(Suppl. 18):S116-S134

9. Sculean A, Bastendorf KD, Becker C, et al. A paradigm shift in mechanical biofilm management? Subgingival air polishing: a new way to improve mechanical biofilm management in the dental practice. Quintessence Int. 2013;44(7):475-477.

10. Hägi T, Hofmänner P, Eick $S$, et al. The effects of erythritol air-polishing powder on microbiologic and clinical outcomes during supportive periodontal therapy: Six-month results of a randomized controlled clinical trial. Quintessence Int. 2015;46:31-41.

11. Flemmig TF, Hetzel $M$, Topoll $H$, et al. Subgingival debridement efficacy of glycine powder air polishing. J Periodontol. 2007;78(6):1002-1010.

12. Hägi TT, Hofmänner P, Salvi GE, Sculean A, Ramseier CA. Clinical outcomes following subgingival application of a novel erythritol powder by means of air-polishing in supportive periodontal therapy. A randomized, controlled clinical study. Quintessence Int. 2013;44:753-761. 
13. Dragoo M. A clinical evaluation of hand and ultrasonic instruments on subgingival debridement. 1. With unmodified and modified ultrasonic inserts. Int J Periodontics Restorative Dent. 1992;12(4):310-323.

14. Checchi L, Forteleoni G, Pelliccioni GA, Loriga G. Plaque removal with variable instrumentation. J Clin Periodontol. 1997;24:715-717.

15. World Workshop in Clinical Periodontics. Nevins M, Becker W, Kornman K (eds), Proceedings of the World Workshop in Clinical Periodontics : Princeton, New Jersey, July 23-27, 1989. Chicago, IL: American Academy of Periodontology;1989.

16. Scapoli C, Mamolini E, Trombelli L. Role of IL-6, TNF-A and LT- A variants in the modulation of the clinical expression of plaque- induced gingivitis. J Clin Periodontol. 2007;34:1031-1038.

17. Pretty IA, Edgar WM, Smith PW, Higham SM. Quantification of dental plaque in the research environment. J Dent. 2005;33(3):193-207.

18. Botti RH, Bossù M, Zallocco N, Vestri A, Polimeni A. Effectiveness of plaque indicators and air polishing for the sealing of pits and fissures. Eur J Paediatr Dent. 2010;11(1):15-18.

19. da Silva $\mathrm{CH}$, Paranhos HF. Efficacy of biofilm disclosing agent and of three brushes in the control of complete denture cleansing. J App Oral Sci. 2006;14(6):454-459.

20. Montevecchi M, Checchi V, Gatto MR, Klein S, Checchi L. The use of a disclosing agent during resective periodontal surgery for improved removal of biofilm. Open Dent J. 2012;6:46-50. Epub 2012 Feb 6.

21. Jayanthi M, Shilpapriya M, Reddy VN, et al. Efficacy of three-tone disclosing agent as an adjunct in caries risk assessment. Contemp Clin Dent. 2015;6:358-363.

22. Crawford AN, McAllan LH, Murray JJ, Brook AH. Oral hygiene instruction and motivation in children using manual and electric toothbrushes. Community Dent Oral Epidemiol. 1975;3(6):257-261.

23. Silva DD, Gonçalo Cda S, Sousa Mda L, Wada RS. Aggregation of plaque disclosing agent in a dentifrice. J Appl Oral Sci. 2004;12(2):154-158.

24. Teitelbaum AP, Pochapski MT, Jansen JL, et al. Evaluation of the mechanical and chemical control of dental biofilm in patients with Down syndrome. Community Dent Oral Epidemiol. 2009;37:463-467.

25. Smith RN, Brook AH, Elcock $C$. The quantification of dental plaque using an image analysis system: reliability and validation. J Clin Periodontol. 2001;28:1158-1162.

26. Smith RN, Rawlinson A, Lath D, et al. Quantification of dental plaque on lingual tooth surfaces using image analysis: reliability and validation. J Clin Periodontol. 2004;31:569-573.
27. O'Leary TJ, Drake RB, Naylor JE. The plaque control record. J Periodontol. 1972;43(1):38.

28. Petersilka GJ, Tunkel J, Barakos K, et al. Subgingival plaque removal at interdental sites using a low-abrasive air polishing powder. J Periodontol. 2003;74(3):307-311.

29. Petersilka GJ, Steinmann D, Häberlein I, Heinecke A, Flemmig TF. Subgingival plaque removal in buccal and lingual sites using a novel low abrasive air-polishing powder. J Clin Periodontol. 2003;30:328-333.

30. Petersilka G, Heckel R, Koch R, Ehmke B, Arweiler N. Evaluation of an ex vivo porcine model to investigate the effect of low abrasive air polishing. Clin Oral Investig. 2018;22:2669-2673.

31. Bozbay E, Dominici F, Gokbuget AY, et al. Preservation of root cementum: a comparative evaluation of power-driven versus hand instruments. Int J Dent Hyg. 2018;16:202-209.

32. Wennström JL, Dahlén G, Ramberg P. Subgingival debridement of periodontal pockets by air polishing in comparison with ultrasonic instrumentation during maintenance therapy. J Clin Periodontol. 2011;38:820-827.

33. Chounchaisithi N, Santiwong B, Sutthavong S, Asvanit P. Use of a disclosed plaque visualization technique improved the self-performed, tooth brushing ability of primary schoolchildren. J Med Assoc Thai. 2014;97:S88-S95

34. Peng Y, Wu R, Qu W, et al. Effect of visual method vs plaque disclosure in enhancing oral hygiene in adolescents and young adults: a single-blind randomized controlled trial. Am J Orthod Dentofacial Orthop. 2014;145(3):280-286.

35. Carter K, Landini G, Walmsley AD. Au tomated quantification of dental plaque accumulation using digital imaging. J Dent. 2004;32:623-628.

36. Chapple IL, Van der Weijden F, Doerfer C, et al. Primary prevention of periodontitis: managing gingivitis. J Clin Periodontol. 2015;42:S71-S76.

How to cite this article: Mensi M, Scotti E, Sordillo A, Agosti $\mathrm{R}$, Calza S. Plaque disclosing agent as a guide for professional biofilm removal: A randomized controlled clinical trial. Int $J$ Dent Hygiene. 2020;00:1-10. https://doi.org/10.1111/ idh.12442 